\begin{tabular}{|c|l|}
\hline Title & U pper boundary of the Pacific plate subducting beneath Hokkaido, Japan, estimated from ScSp phase \\
\hline Author(s) & Osada, Kinue; Y oshizawa, Kazunori; Y omogida, Kiyoshi \\
\hline Citation & $\begin{array}{l}\text { Physics of the Earth and Planetary Interiors, 183(1-2), 63-72 } \\
\text { https://doi.org/10.1016/.pepi.2010.06.006 }\end{array}$ \\
\hline Issue Date & 2010-11 \\
\hline Doc URL & http://hdl.handle.net/2115/44908 \\
\hline Type & article (author version) \\
\hline File Information & PEPI183-1-2_63-72.pdf \\
\hline
\end{tabular}

Instructions for use 


\title{
Upper Boundary of the Pacific Plate Subducting Beneath Hokkaido, Japan, Estimated from $S c S p$ Phase
}

\author{
Kinue Osada ${ }^{\mathrm{a}, 1}$, Kazunori Yoshizawa ${ }^{\mathrm{a}, \mathrm{b}}$, Kiyoshi Yomogida*,a \\ ${ }^{a}$ Earth and Planetary Dynamics, Faculty of Science, Hokkaido University, Sapporo \\ 060-0810, Japan \\ ${ }^{b}$ Lamont-Doherty Erath Observatory, Columbia University, Palisades, NY 10964, USA
}

\begin{abstract}
Three-dimensional geometry of the upper boundary of the Pacific plate subducting beneath Hokkaido, Japan, was obtained using the $S c S p$ phase: the phase converted from $S c S$ (S wave reflected at the core-mantle boundary) to $\mathrm{P}$ wave at the plate boundary. Taking the advantage of a dense seismic network, "Hi-net", recently deployed across the Japanese islands, we applied several seismic array analyses to the recorded waveform data for a large nearby deep earthquake, in order to enhance very weak $S c S p$ signals in the original records. At first, we set up five blocks for the region in plate dip directions. After aligning the travel times of $S c S$ and stacking seismograms among stations in a given sub-block perpendicular to each dip direction, we searched for the optimal plate model (i.e., two-dimensional geometry of the upper boundary) for each block. The model was parameterized by seven depth grids, and seismograms were stacked based on the travel time of $S c S p$ as a time lag of each sub-block, so that the optimal model would yield the
\end{abstract}

\footnotetext{
* Corresponding author

Email address: yomo@mail.sci.hokudai.ac.jp (Kiyoshi Yomogida)

${ }^{1}$ Now at: NEC Aerospace Systems, Sapporo Branch, Sapporo 060-0808, Japan
} 
maximum spectral energy of $S c S p$ after stacking. This model parameter search was conducted, using ray tracings of $S c S p$ with a reference velocity model and a non-linear inversion scheme (Neighbourhood Algorithm). The optimal model of each block was combined each other by cubic spline interpolation, in order to construct an overall three-dimensional geometry of the upper boundary of the plate. Next, we performed the frequency-wavenumber $(f-k)$ spectral analysis to refine the above result. Assuming each station as a reference point, we made beam output from records of its adjacent stations as a function of wavenumber vector $\left(k_{x}, k_{y}\right)$ and frequency. The peak of its power spectrum was considered to represent the wavenumber vector of $S c S p$, that is, azimuth of arrival and slowness, so that we can estimate the position and depth of the corresponding $S c S-S c S p$ conversion. In the frequency range from 0.5 to $1.5 \mathrm{~Hz}$, we could estimate the conversion points for 21 stations or hypothetical arrays, and revised the geometry of the upper boundary obtained by the non-linear stacking approach in the previous step. The final plate model was compared with the distribution of intraplate earthquakes in the Pacific plate. This comparison clearly reveals that the upper seismic zone merges with the lower from 150 to $200 \mathrm{~km}$ in depth, deviating systematically away from the upper boundary where the boundary is slightly bumped in a convex manner.

Key words: $\quad S c S p$ phase; subducting slab; Pacific plate; upper boundary; array processing 


\section{Introduction}

Regions around subducting plates or slabs are recognized to be highly anomalous in the propagation of seismic waves for a long time. Tomographic studies have been widely conducted in subduction zones over the world to determine large-scale spatial distribution of P and/or S-wave velocities (e.g., Fukao et al., 2001). These studies with travel-time data are, however, not suitable to retrieve sharp discontinuities or boundaries in velocity. There are several seismic approaches appropriate to structural boundaries like upper boundary of a subducting plate. The upper boundary is an important, sharp discontinuity of the earth's interior, due to its dominant role over various geodynamic activities in a subduction zone. It has been studied extensively using focal mechanisms in slab, reflected and/or refracted waves for local or artificial events, and receiver function analysis for teleseismic events. Nevertheless, one of the most straightforward and effective seismic approaches to focus on the profile of the upper plate boundary is the use of $S c S p$ phase.

$S c S p$ is the wave observed as a precursor of $S c S$ that is reflected from the core-mantle boundary (CMB) and then propagates upwards nearly vertically. It is converted from $\mathrm{S}(S c S)$ to $\mathrm{P}$ wave at the upper boundary. In comparison with $S c S$, the measurement of $S c S p$ eliminates all the complicated effects such as source, station site and lateral heterogeneities other than the upper plate boundary, so that the value of boundary depth can be estimated directly with good precision. Okada (1979) was the first attempt to profile a subducting plate with the $S c S p$ phase observed in Hokkaido, Japan. Several researches on $S c S p$ phases have been followed in other parts of the world (e.g. Snoke et al, 1977; Helffrich et al., 1989; Helffrich and Stein, 1993; Helffrich, 1996; 
Bourne and Stuart, 2002).

In Japan, $S c S p$ phases are observed clearly in some areas, and have been used to estimate the depth profile of upper plate boundaries even before the deployment of the high-density seismic network, Hi-net, in late 1990s: for example, in the Chugoku-Shikoku region by Nakanishi (1980) and in the Kanto region by Iidaka and Obara (1993). Thanks to the recent deployment of high-density seismic networks over the world resembling the Hi-net system in Japan (Obara, 2003), it is possible to obtain a detail trajectory in depth of the upper boundary of a subducting plate. Umino et al. (2002) obtained the overall depth distribution of subducting plates in and around Japan, using the Hi-net data.

In contrast, the $S c S p$ phase has not been observed clearly in the Hokkaido region, except for some limited areas close to the trench axis (e.g., the station KMU located at Cape Erimo, as observed by Okada (1979)). Compared with other regions in Japan, reliable estimation of the spatial distribution of the upper boundary of the Pacific plate subducting there has been a difficult issue. For example, while receiver function analyses have imaged various boundaries clearly in other parts of Japan (e.g. Shiomi et al., 2004; Tonegawa et al., 2006; Kawakatsu and Watada, 2007; Shiomi et al., 2008), such images in the Hokkaido region have never been obtained except for a depth range shallower than $100 \mathrm{~km}$ (Hirahara et al., 2005). This difference may be related to some specific features in this region, for example, (1) the trench axis of the Pacific Ocean is farther from the coast of the island arc than the other areas in Japan, so that the upper boundary beneath stations on land is relatively deep, resulting in very weak $S c S p$ signals, (2) the majority of 
Hokkaido is covered with soft and relatively thick surface layers, generating high levels of background noise that prevent the detection of weak $S c S p$ signals, and (3) the upper boundary of the Pacific plate beneath in the Hokkaido region seems to be not as sharp as tjat beeath the other regions of Japan, making the amplitude of $S c S p$ phases relatively small. For similar reasons, the seismicity related to the Pacific plate subducting in the Hokkaido region has also been investigated the least in Japan, in spite of dense seismic networks. Katsumata et al. (2003) was the first systematic search to obtain a reliable three-dimensional distribution of earthquakes associated with the Pacific plate in the Hokkaido region, utilizing the temporary deployment of many new stations in addition to Hi-net stations.

This study aims to estimate three-dimensional geometry of the upper plate boundary (or a suite of two-dimensional depth distribution) with $S c S p$ phases recorded with Hi-net stations in Hokkaido. Since individual $S c S p$ phases recorded in this region are very weak, we enhance their signals with several array processing techniques such as slant stacking, non-linear inversion with ray tracing, and frequency-wavenumber spectral analysis, to estimate the $S c S$ to $P$ conversion depth at a given small area. We shall compare our results with the seismic event distribution obtained by Katsumata et al. (2003), and discuss the spatial seismic pattern and its related stress field in the Pacific plate subducting in the Hokkaido region.

\section{Data and Pre-processing}

We collected the seismograms recorded at the total of 112 stations in Hokkaido as a part of the seismic network, Hi-net, deployed over the Japanese 
islands (Obara, 2003). It is desirable that an impulsive $S c S$ wave of large amplitude is incident to the upper plate boundary upwards as vertically as possible for our purpose. After the establishment of Hi-net, the largest deep event in this region occurred on 17 November 2002 in the south of the Sea of Okhotsk at the focal depth of $476.8 \mathrm{~km}$ with $M w=7.3$. We used seismic records from this event. The distribution of the stations used in this study is shown in Figure 1. The focal mechanism in this figure is taken from the Global CMT catalog (Ekström et al., 2005).

In preprocessing of the present data, we removed instrumental response of the observed three-component velocity waveform records, and transformed them into displacement data. The obtained horizontal two-component data were rotated into radial and transverse components, followed by their bandpass filtering between 0.1 and $0.5 \mathrm{~Hz}$ for the stacking analysis presented in the next section and between 0.5 and $1.5 \mathrm{~Hz}$ for the following $f$ - $k$ spectral analysis. As explained later, the spatial extent of stations for the stacking analysis is much larger than that of the $f-k$ spectral analysis, resulting in a lower frequency filter in the former case. $S c S$ phases are clearly identified at this stage for all the seismograms.

Since these $S c S$ waves were radiated from the source in a very limited range of azimuth, we can ignore all the complicated source effects, path effects between the source and the upper plate boundary and site effects by shifting all the $S c S$ phases to be aligned in time. The $S c S$ phase at the station SMPH is the clearest of all, setting it as the reference station. We take its cross-correlations with other $S c S$ waveforms, shifting seismograms in time to achieve the maximum correlations. In other words, we arrange 
seismograms as if the $S c S$ phase arrives simultaneously at each station. In order to avoid any possible anisotropic effects, we utilized radial-component $S c S$ phases because we shall focus on the converted $S c S p$ phases in the vertical component in the subsequent analyses. After the above shift of seismograms in time, we use vertical seismograms in which the amplitudes of $S c S p$ are the largest.

\section{Stacking analysis and reconstruction of slab geometry}

\subsection{Slant stack analysis}

Very weak $S c S p$ signals recorded in this region prevent us from any standard analyses and inversion schemes to estimate the image of the plate boundary. Since the overall geometry of the subducting Pacific plate is known in this region, we first divide the studied region into several rectangular blocks in the plate dip direction. Then, we attempt to obtain the 2-D geometry of the upper plate boundary in each block, using the slant-stack technique of seismograms at stations located in a given block. Figure 2 shows an example of the present analysis. Along the dip direction of the Pacific plate, we take a block of $90 \mathrm{~km}$ wide, dividing it into sub-blocks perpendicular to the original block (i.e., along the trench axis) of $20 \mathrm{~km}$ wide. Assuming that the conversion points from $S c S$ to $P$ wave at the upper plate boundary share a common depth, the $S c S p$ phase arrives simultaneously at all the stations within a given sub-block. We simply stack all the time-domain waveforms in a sub-block to enhance $S c S p$ signal, resulting in a representative $S c S p$ waveform at each sub-block, as shown in Figure 2b. The resulted $S c S p$ phase is indeed slightly clearer than that in original waveforms. 
We then apply the slant stack technique to the already stacked waveforms in each sub-block. In a given block, a plane $S c S p$ wave is assumed to arrive at a fictitious station in the center of each sub-block. Then, the $S c S p$ signal should be represented by the stack of these seismograms with a constant slowness $p$, or with a time lag proportional to the distance from a reference point. We shift a seismogram of the $j$-th sub-block with the distance $D_{j}$ from the reference point by $\tau_{j}=D_{j} \cdot p$. We stack seismograms in the slowness range between $-12 \mathrm{~s} / \mathrm{km}$ and zero (e.g., Kawakatsu and Niu, 1994). In this study, we employ $N$ th-root stacking (Kanasewich, 1973) in order to enhance weak $S c S p$ signals as much as possible. The stacked value $y_{i}(p)$ at time $i$ is defined by

$$
y_{i}(p)=R_{i}(p)\left|R_{i}(p)\right|^{N-1}
$$

where

$$
R_{i}(p)=\frac{1}{K} \sum_{j=1}^{K} \operatorname{sgn}\left(w_{j}\left(i+\tau_{j}\right)\right)\left|w_{j}\left(i+\tau_{j}\right)\right|^{1 / N}
$$

$w_{j}(i)$ is the amplitude of the waveform of the $j$-th sub-block at time $i, K$ is the number of sub-blocks in the given block, and sgn represents the sign of a value (i.e., +1 for a positive value, -1 for a negative value and 0 for zero). The above result in the case of $N=1$ is reduced to a simple linear stack. The optimal value of $N$ that enhances signals relative to noise depends on the quality of data, particularly how much coherent components are contaminated in noise. After several tests with various values of $N$, we found that $S c S p$ is the most prominent with our present data set in the case of $N=2$.

Stacking seismograms with various values of slowness $p$ as explained above (Figure 2b), we obtain a diagram of the stacked waveforms in the timeslowness domain. We plot their envelopes in this diagram, using the Hilbert 
transform of the stacked waveforms (Figure 2c). The maximum in this figure should correspond to the travel-time and slowness of the $S c S p$ phase in the given block. In this example, we obtain $t=-25.0 \mathrm{sec}$ and $p=-7.25 \mathrm{~km} / \mathrm{deg}$. We thus estimate the arrival time and slowness of the $S c S p$ phase relative to the $S c S$ phase in each block, assuming that the angle of the corresponding part of the subducting plate is constant. With reasonable depth distributions of $\mathrm{P}$ and $\mathrm{S}$ waves above the upper plate boundary, we can pinpoint the depth and horizontal location of the conversion point from $S c S$ to $P$ phase. These results for five blocks in the studied region are summarized in Figure 3. All the maxima in the time-slowness diagram are quite clearly identified, so that we confirm that the present waveforms contain sufficient energy of the $S c S p$ phase although we cannot find such signals clearly in the original seismograms.

\subsection{Non-linear parameter search for slab geometry}

The slant-stack approach assumes that a plane $S c S p$ wave is incident at stations in each block. This assumption may be too brave because station locations in a given block are spread over $200 \mathrm{~km}$ along its dip direction. We therefore introduce a new approach to determine 2-D geometry of the upper boundary in each block, by stacking seismograms with $S c S p$ with dip angle of the boundary to vary freely. We search for the optimal 2-D geometry of the upper boundary by stacking $S c S p$ signals based on this flexible slab geometry. The shape of each 2-D upper plate boundary is expressed by cubic spline functions with discrete grid points along the plate (Figure 4a). We trace rays of $S c S p$ phase with the assumed plate geometry to compute ray-theoretical travel time at the reference point of each sub-block. Strictly 
speaking, it is the time lag from $S c S$ phase. Based on the computed time lags, we stack all the seismograms (i.e., already pre-stacked within each sub-block) in a given block, searching for the maximum of the stacked seismograms that should correspond to the optimal plate geometry. This procedure is highly non-linear so that we estimate the optimal model parameters with the Neighbourhood Algorithm, called NA hereafter (Sambirdge, 1999).

For each profile of the upper boundary along the dip of the Pacific plate (i.e., beneath each block of Figure 3), we assign seven points of 100, 200, $300,400,500,600$ and $800 \mathrm{~km}$ in depth away from the trench axis as model parameters, which forms the 2-D geometry of the upper boundary by a cubic spline function (Figure 4a). For an assigned geometry (called a plate model hereafter), we trace rays of $S c S p$ for a plane $S c S$ wave incident from below vertically. As shown in Figure 1, epicentral distances in this study are less than eight degrees. Even though $S c S$ is radiated from a point-like source, the above assumption (i.e., vertical plane-wave incidence) is sufficiently valid, because the slowness of $S c S$ at each station is as small as $1 \mathrm{sec} / \mathrm{deg}$.

The velocity model ak135 (Kennett et al., 1995) in Figure 4b is used, by adding a velocity discontinuity of $10 \%$ at the plate boundary. The uncertainty of this velocity jump should affects the amplitude of $S c S p$ or the conversion rate from $S c S$ to $P$ but not their travel time difference, which is essential in this study. With the dip angle of 30 degrees, for example, the amplitude ratio of $S c S p / S c S$ is changed from 0.07 to 0.12 for velocity contrast at the boundary from 5 to $10 \%$ while the travel time difference by less than 1 sec. With a steeper dip angle, the amplitude ratio changes a little, so as the travel time difference. Since the dip angle of the Pacific plate in 
the Hokkaido region is about 30 degrees, our study to retrieve the depth profile of the upper boundary, based on not amplitude ratio but difference in travel time, should not be affected by the choice of the velocity contrast. (See a rather critical case in contrast with a nearly horizontal dip angle in the Shikoku-Chugoku, Japan, region in Nakanishi (1980)). We employed a standard ray-tracing scheme for a 1-D velocity structure with one discontinuous boundary (i.e., the upper plate boundary). Based on the computed travel times of the $S c S p$ phase incident to sub-blocks of each block, we stack all the seismograms in the block, similar to the slant stack in the previous section.

For the optimal plate model, the stacked $S c S p$ phase should have the maximum amplitude in the model space. The amplitude may vary in a complicated or non-linear manner with parameters of the plate model, so that we adopt the neibourhood algorithm (NA). A highly non-linear problem may be difficult to solve because we must combine two conflicting factors: random sampling of possible model parameters and efficient iterative scheme to converge into the optimal model. The criterion to balance these two factors may tend to be subjective or a priori, including the present NA method. The main parameters for such a criterion in NA are the following two numbers: (1) $N$, the number of models generated in each iteration step of model parameter search and (2) $M$, the number of optimal models that lead to new $N$ models in the subsequent iteration step. After several tests with various values of these two tunable parameters, we found that the case with $N=30$ and $M=10$ yielded good results in our present data set and modeling.

Figure 5 shows an example of the results of the NA inversion, for block 
2 in Figure 3. The top of Figure 5 shows the total of 6,500 plate models generated during the model parameter search with the NA. For each plate model, we computed the travel time difference between $S c S$ and $S c S p$ phases at each sub-block, then stacked all the waveforms by each time difference, as shown in the bottom of Figure 5. We selected the best $M$ models from $N$ models for the subsequent iteration step, using a criterion with the spectral energy of the stacked seismograms in the time window of a possible $S c S p$ arrival. After such iteration steps, the plate model to give the maximum $S c S p$ energy was judged to be the best. Figure 6 shows the best plate models for the five blocks in Figure 3, together with rays of $S c S p$ phases. Compared with the preliminary results of the slant-stack analysis with the plate model of constant dip angles in the previous section, irregular undulations at plate boundaries are apparent with these models, implying the necessity of a detailed 2-D geometry for each block along the dip direction, to explain our present $S c S p$ data. We combine the plate models for the five blocks with a cubic spline function in horizontal directions along the trench axis, resulting in the 3-D plate model shown in Figure 7. This figure includes ray paths of $S c S p$ for block 4 .

\section{4. $f$ - $k$ Spectral Analysis}

The stacking analysis in the previous section may be appropriate to obtain the overall geometry of the upper plate boundary in this region, because the number of stations is still not sufficient to identify its detailed irregularity, particularly we needed to stack waveforms at several stations of a given sub-block into one data set to be inverted. We also introduced five 2-D plate 
models along dip directions. Station pairs are apart more than $200 \mathrm{~km}$ in some cases for which good cross-correlation may not be expected due to complex subsurface structure of this region (Katsumata et al., 2003). Since we can only estimate a 2-D profile of conversion points (i.e., depth and location) in each case, more accurate and reliable results on the upper boundary may be obtained in the use of an additional type of array-type approaches. Here, we apply the frequency-wavenumber spectral analysis (called $f-k$ analysis), assuming a seismic array consisting of a given station and its adjacent stations (e.g., Capon, 1973; Aki and Richards, 1980). As schematically shown in Figure 8, we set one reference station and its adjacent stations, assuming that a plane wave is incident locally with wavenumber vector $\left(k_{x}, k_{y}\right)$ and angular frequency $\omega$. Changing the values of wavenumber vector components, we search for the maximum power spectrum by taking their cross-correlations at each frequency. The spatial extent of each array is about $50 \mathrm{~km}$, so that the assumption of a constant slowness within an array is valid, while we allow slowness to vary from array to array, depending on the 3-D geometry of the upper boundary.

With the travel time $t_{0}$ of a specific wave $(S c S p$ phase in this case) at the reference station of $\left(x_{0}, y_{0}\right)$, the time shift $t_{i}$ for the record at the $i$-th station located at $\left(x_{i}, y_{i}\right)$ at frequency $\omega$ may become

$$
t_{i}=t_{0}+\frac{k_{x}}{\omega}\left(x_{i}-x_{0}\right)+\frac{k_{y}}{\omega}\left(y_{i}-y_{0}\right) .
$$

By summing the total of $N$ records $w_{i}(t)$ at the $i$-th station by the time shift $t_{i}$ in equation (3), we get the following beam output:

$$
b\left(k_{x} / \omega, k_{y} / \omega, t\right)=\frac{1}{N} \sum_{i=1}^{N} w_{i}\left(t+t_{i}\right) .
$$


The $f$ - $k$ power spectrum is the Fourier transform of the auto-correlation (denoted by $<>$ ) of beam outputs:

$$
P\left(k_{x}, k_{y}, \omega\right)=\int_{-\infty}^{\infty} d \tau \cdot \exp (i \omega \tau)\left\langle b\left(k_{x} / \omega, k_{y} / \omega, t\right) \cdot b\left(k_{x} / \omega, k_{y} / \omega, t+\tau\right)\right\rangle .
$$

At a given frequency $\omega$, the maximum of these power spectra in the wavenumber domain gives the slowness and back-azimuth of the arriving phase as follows:

$$
p=\frac{\sqrt{k_{x}^{2}+k_{y}^{2}}}{\omega} \text { and } \theta=\tan ^{-1}\left(k_{x} / k_{y}\right)
$$

In this study, we assume each of all the 112 stations as a reference station with its adjacent stations in the radius of $50 \mathrm{~km}$. The average number of stations for each array is about seven. Figure 9 shows the results of the $f$ - $k$ spectral analysis for seven example reference stations in the frequency range from 0.5 to $1.5 \mathrm{~Hz}$. The peak of power spectra in the $\left(k_{x}, k_{y}\right)$ domain should correspond to the $S c S p$ phase arriving at each array, and we can estimate its slowness and back-azimuth from its wavenumber vector by equation (6). The dotted lines in the right of Figure 9 represent the arrival times of the maximum beam output, which should correspond to $S c S p$ phases.

Although we attempted to identify the spectral peaks for all the 112 stations in a wide range of frequencies, we could obtain the limited number of successful cases. Correlations among waveforms within each array are poor in most of cases, probably because the signals of $S c S p$ phases in this area are indeed very weak and the average radius of arrays (about $50 \mathrm{~km}$ ) is relatively large, that is, the spatial density is still not sufficient even for the present distribution of Hi-net stations. Correlations at low frequency are relatively high, as shown in Figure 9, but the signals of $S c S p$ are weak, while low 
correlations even with apparent large signals at high frequency. As a result, we could identify clear spectral peaks (i.e., $S c S p$ phase) for 21 stations in the frequency range of Figure 9, that is, from 0.5 to $1.5 \mathrm{~Hz}$. In these successful cases, we projected the ray path from a given station backwards, using the estimated wavenumber vector $\left(k_{x}, k_{y}\right)$ or $p$ and $\theta$ with the velocity model ak135, similar to the stacking analysis. By the travel-time difference from that of the $S c S$ phase, we estimated the depth and location of the conversion point of the $S c S p$ phase (i.e., the upper plate boundary) for each station or array.

Since the number of the estimated depths and locations of the upper plate boundary is limited with our present data set, we could not make a complete 3-D map of the boundary in a similar manner to the standard seismic tomography. Since we already obtained a plate model by the stacking-NA approach in the previous section, we used the results of the $f-k$ analysis to revise this plate model. The left of Figure 10 shows the contours of the depth distribution of the plate model derived from the NA inversion in the previous section with the 21 conversion points (i.e., the upper plate boundary) estimated by the $f$ - $k$ analysis. While the previous stacking analysis provides the overall geometry of the plate model, the present $f-k$ analysis gives more accurate estimation of its depths and locations although we can only obtain such values at the limited number of points. In other words, these two results are complimentary, so that we obtain a reliable and accurate final plate model in this region from the combination of the two independent analyses. The right of Figure 10 shows the cross section of the final upper plate boundary in the rectangular section of the left, together with its event locations (Katsumata 
et al., 2003).

The present final result does not differ significantly from previous works such as Umino et al. (2002). Nevertheless, it may be the first opportunity that we can compare the plate model (i.e., the upper boundary) with the associated seismicity for the Pacific plate in the Hokkaido region where both plate structure and seismicity have been least investigated in Japan, due to the difficult factors mentioned in the Introduction. Katsumata et al. (2003) revealed a double seismic zone in the plate down to the depth of about 150 $\mathrm{km}$, then the two zones appear to be converged below this depth. The right of Figure 10 clearly supports that the upper seismic zone shifts into the lower zone systematically, departing from the upper plate boundary in this depth range. The upper boundary at the depth of about $200 \mathrm{~km}$ is slightly bumped upwards in a convex manner. One may claim velocity anomaly overlying this part of the slab to be responsible for the above apparent localized bump in plate geometry. If it were the case, a part of extremely negative anomaly in $V s / V p$ would be required, because of the present use of the travel time difference between $S c S p$ and $S c S$. Such an anomaly might be possible at a very localized part of mantle wedge. Nevertheless, tomographic study by Katsumata et al. (2006) did not detect any large anomalies in terms of $V s / V p$ that would affect the local depth profile of the slab, as we obtained in this study. Complex flexure of a slab has been reported in several areas, for example, the Philippines Sea plate in southwest Japan, as studied in detail by Shiomi et al. (2004, 2008); Tonegawa et al. (2009). Implying its structural effect on the variation of event locations shown in Figure 10, relationship between stress state and possible factors to generate deep seismic events 
should be extremely important in this not-well investigated subduction zone.

\section{Conclusions}

Applying several array processing techniques to the Hi-net data with a very large deep adjacent event, we obtained the $3-\mathrm{D}$ geometry or the 2-D distribution of the upper boundary of the Pacific plate subducting beneath Hokkaido, Japan. We attempted to enhance the signals of $S c S p$ phase converted from $S c S$ phase at the upper boundary although original $S c S p$ phases in record are too weak to be identified.

We first confirmed the existence of $S c S p$ signals in our data set by simple slant-stacking analysis for blocks in dip directions, assuming a constant dip angle in each block. Introducing general 2-D profiles of the upper boundary, we calculated ray paths of $S c S p$ phases for vertically upgoing plane $S c S$ waves. The travel time (i.e., time lag) was computed for $S c S p$ relative to $S c S$ at each sub-block with these rays, and records were stacked with the time lag. The optimal 2-D profile of the upper boundary was then inverted for each block, using the Neibourghhood Algorithm (NA) for this highly non-linear problem. Combining the results of all the blocks, we established the overall 3-

D geometry of the upper plate boundary in this region. In order to utilize the advantage of the dense Hi-net data as seismic arrays, we further conducted $f$ $k$ spectral analysis for arrays composed of a reference station and its adjacent stations. We searched for the maximum of beam output in the wavenumber domain for the slowness and back azimuth of the arriving $S c S p$ phase at each array. In spite of difficult observational conditions in this region, we could identify $S c S p$ signals for 21 stations or arrays in the frequency range 
from 0.5 to $1.5 \mathrm{~Hz}$. Tracing a ray backwards with the estimated slowness and back azimuth at each array and using the time lag of each $S c S p$ phase, we estimated the depth and location of the $S c S-S c S p$ conversion point, that is, the upper plate boundary. This procedure revised the 3-D geometry of the plate model obtained in the previous step. In a cross section across the center of Hokkaido, we found close relationship between the seismicity in the double seismic zone and the irregularity of the upper plate boundary from 150 to $200 \mathrm{~km}$ depth.

The Hi-net in Japan is still one of the best local seismic arrays over the world for their quality, density, aperture and uniformity in data (Obara, 2003). The subducting plates have been investigated in detail by a number of recent researches, including seismic tomography (Zhao et al., 1994; Nakajima et al., 2001) and receiver-function analysis (Shiomi et al., 2004; Tonegawa et al., 2006; Kawakatsu and Watada, 2007). Although these studies proposed many interesting features of slab dynamics in and around Japan, investigations of the structure associated with the Pacific plate subducting in the Hokkaido region have not been successful yet (Hirahara et al., 2005). Array analyses such as those presented in this study are essential to obtain the image of the upper plate boundary, the strongest structural boundary, in this specific subduction zone of Japan, which should be also useful to many subduction zones in the world.

Even with the present Hi-net data, we could utilize cross-correlations among the observed seismograms only in a relatively low frequency range, say, lower than $1 \mathrm{~Hz}$. We cannot therefore expect good spatial resolution of the image of the plate boundary with $S c S p$ phases. Since the present signal 
level was not sufficient for $S c S p$ phases even after stacking, we could only use their time lags but not other kinds of $S c S p$ information, such as the amplitude ratio of $S c S p / S c S$ and its frequency dependency, which would lead us to quantitative estimations of physical properties of the upper plate boundary. For example, if data from several deep adjacent events are available, we may stack records of not only neighbouring stations but also all the events in order to obtain large $S c S p$ signals. Collecting data for more deep large adjacent events in this region and introducing more effective array processing methods, we may enhance $S c S p$ signals to retrieve any additional pieces of information on the physical states of the upper plate boundary in this seismically active and tectonically important region in the future.

\section{Acknowledgments}

The waveform data used in this study were provided by the Institute of Seismology and Volcanology (Hokkaido University) and Japan Meteorological Agency. Part of the data was downloaded from the website of Hi-net operated by the National Research Institute for Earth Science and Disaster Prevention of Japan. We thank Malcolm Sambridge for the provision of the NA code, as well as Dr. G. Helffrich and an anonymous reviewer for their many useful comments. This study was partly supported by Grant-in-Aid for Scientific Research (No. 16075201) from the Ministry of Education, Culture, Sports, Science and Technology of Japan. Maps and diagrams were generated using the Generic Mapping Tools (Wessel and Smith, 1998), 


\section{References}

Aki, K., and Richards, P.G., 1980. Quantitative Seismology: Theory and Methods, W.H. Freeman, San Francisco, 932 pp.

Bourne, M., and Stuart, G., 2002. ScSp observed on North Island, New Zealand: Implications for subducting plate structure, Geophys. J. Int., 142: $925-232$.

Capon, J., 1973. Signal processing and frequency-wavenumber spectrum analysis for a large aperture seismic array. In: Bolt, B.A. (Ed.), Methods of Computational Physics, 13: 1-59.

Ekström, G., Dziewoski, A.M., Maternovskaya, N.N., and Nettles, M., 2005. Global seismicity of 2002: centroid-moment-tensor solutions for 1034 earthquakes, Phys. Earth Planet. Inter., 148: 303-326.

Fukao, Y, Widiyantoro, R.D., and Ohbayashi, M., 2001. Stagnant slab in the upper and lower mantle transition zone, Rev. Geophys., 39: 291-323.

Helffrich, G., 1996. Subducted lithospheric slab velocity structure: Observations and mineralogical inferences, In: Bebout, G., Scholl, D., Kirby, S., and Platt, J. (Ed.), Subduction Top to Bottom, AGU Geophys. Mon., 96: 215-222.

Helffrich, G., and Stein, S., 1993. Study of the structure of the slab-mantle interface using reflected and converted seismic waves, Geophys. J. Int.,115: 14-40. 
Helffrich, G., Stein, S., and Wood, B.J., 1989. Subduction zone thermal structure and mineralogy and their relationship to seismic wave reflections and conversions at the slab/mantle interface, J. Geophys. Res., 94: 753763.

Hirahara, K., Tonegawa, T., and Shibutani, T., 2005. Receiver function imaging of the crust and the uppermantle structure beneath the Japan Islands, Proc. Seis. Soc. Japan, Fall Meeting, B032.

Iidaka, K., and Obara, K., 1993. The upper boundary of the subducting Pacific Plate estimated from $S c S p$ waves beneath the Kanto region, J. Phys. Earth, 41: 103-108.

Kanasewich, E.R., 1973. Time Sequence Analysis in Geophysics, Univ. Alberta Press Edmonton, 352 pp.

Katsumata, K., Wada, N., and Kasahara, M., 2003, Newly imaged shape of the deep seismic zone within the subducting Pacific plate beneath the Hokkaido corner, Japan-Kurile arc-arc junction, J. Geophys. Res., 108(B12): doi:10.1029/2002JB002175.

Katsumata, K., Wada, N., and Kasahara, M., 2006. Three-dimensional P and S wave velocity structures beneath the Hokkaido corner, Japan-Kurile arc-arc junction, Earth Planets Space, 58: e37-e40.

Kawakatsu, H., and Niu, F., 1994. Seismic evidence for a 920-km discontinuity in the mantle, Nature, 371: 301-305. 
Kawakatsu, H., and Watada, S., 2007. Seismic evidence for deep-water transportation in the mantle, Science, 316: DOI:10.1126/science.1140855, 14681471.

Kennett, B.L.N., Engdahl, E.R., and Buland, R., 1995. Constraints on seismic velocities in the Earth from travel times, Geophys. J. Int., 122: 108124.

Nakajima, J., Matsuzawa, T., Hasegawa, A., and Zhao, D., 2001. Threedimensional structure of $V p, V s$, and $V p / V s$ beneath northeastern Japan: Implications for arc magmatism and fluids, J. Geophys. Res., 106: 2184321857.

Nakanishi, I., 1980. Precursor to $S c S$ phases and dipping interface in the upper mantle beneath southwestern Japan, Tectonophysics, 69: 1-35.

Obara, K., 2003. Hi-net: High-sensitivity seismograph network, Japan. In: Takanami, T., and Kitagawa, G. (Ed.), Methods and Applications of Signal Processing in Seismic Network Operation, Springer Verlag, 79-88.

Okada, H., 1979. New evidences of the discontinuous structure of the descending lithosphere revealed by $S c S p$ phase, J. Phys. Earth, 27: S53-S63.

Sambirdge, M., 1999. Geophysical inversion with a neibourhood algorithm I: Searching a parameter space, Geophys. J. Int., 138, 479-494.

Shiomi K., Sato, H., Obara, K., and Ohtake, M., 2004. Configuration of subducting Philippine Sea plate beneath southwest Japan revealed from 
receiver function analysis based on the multivariate autoregressive model, J. Geophys. Res., 109: B04308, doi:10.1029/2003JB002774.

Shiomi, K., Matsubara, M., Ito, Y., and Obara, K., 2008. Simple relationship between seismic activity along Philippine Sea slab and geometry of oceanic Moho beneath southwest Japan, Geophys. J. Int., 173: 1018-1029.

Snoke, J.A., Sacks, I.S., and Okada, H., 1977. Determination of the subducting lithosphere boundary by use of converted phases, Bull. Seismol. Soc. Amer., 67: 1051 - 1060.

Tonegawa, T., Hirahara, K., Shibutani, T., and Shiomi, K., 2006. Upper mantle imaging beneath the Japan Islands by Hi-net tiltmeter recordings, Earth Planets Space, 58: 1007-1012.

Tonegawa, T., Nishida, K., Watanabe, T., and Shiomi, K., 2009. Seismic interferometry of teleseicmic S-wave coda for retrieval of body waves: an application to the Philippine Sea slab underneath the Japanese Islands Geophys. J. Int., 178: 1574-1586.

Umino, N., Asaon, Y., Okada, T., and Matsuzawa, T., 2002. Geometry of the subducted Pacific slab estimated from $S c S p$ phases observed by the high density seismic network, Proc. Seis. Soc. Japan, Fall Meeting, A50.

Wessel, P., and Smith, W., 1998. New improved version of the generic mapping tools released. EOS Trans. AGU, 79: 579.

Zhao, D., Hasegawa, A., and Kanamori, H., 1994, Deep structure of Japan 
495 subduction zone as derived from local, regional, and telesismic events, J. 496 Geophys. Res., 105: 22313-22329. 


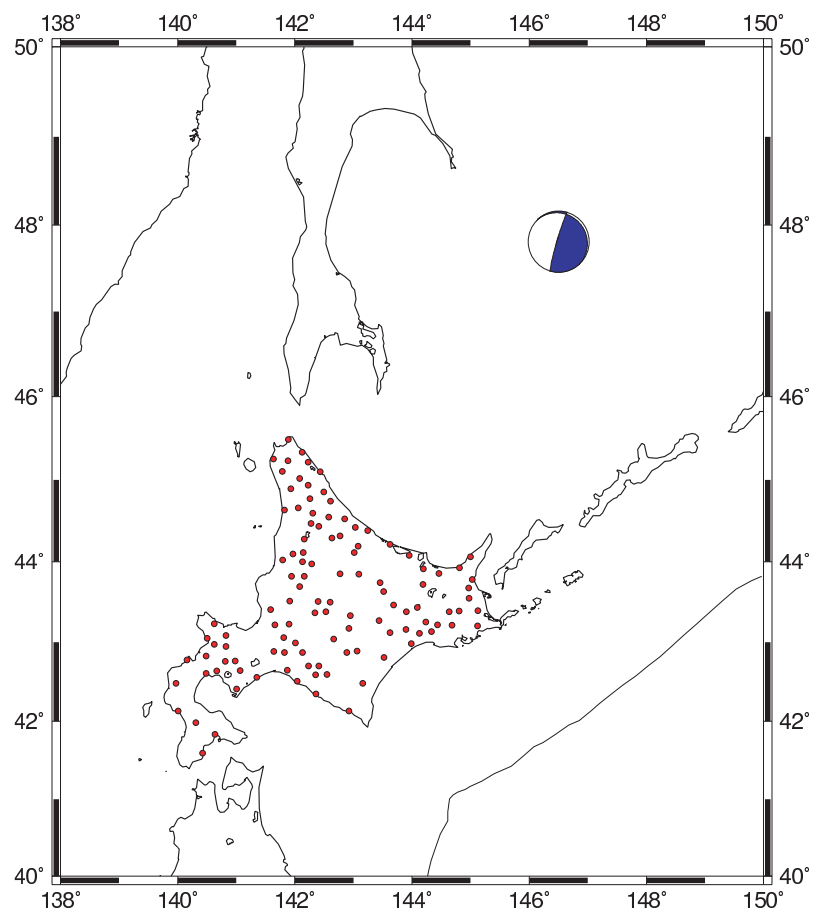

Figure 1: Location of Hi-net stations in Hokkaido, Japan, represented by solid circles, and the epicenter of the used event on 17 November 2002 of $476.8 \mathrm{~km}$ in depth. Focal mechanism from the Global CMT catalog is also shown. 
(a)

(b)

time(s)

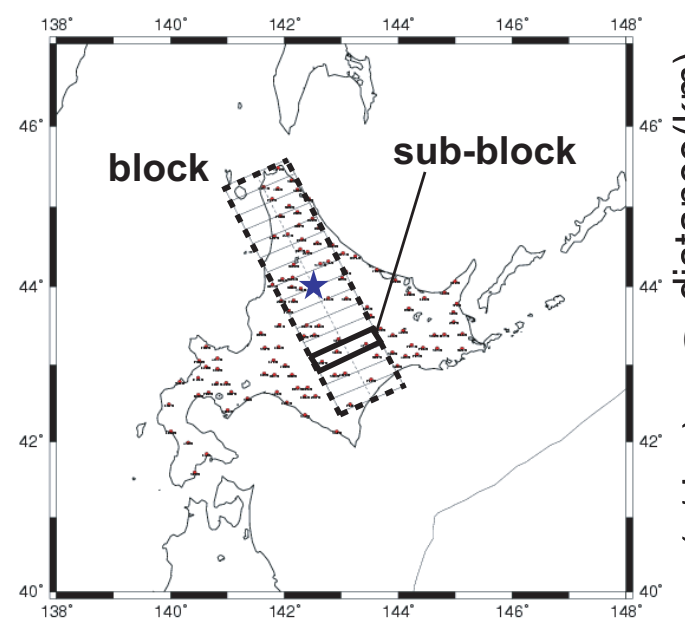

E 200 10-80-70-60-50-40-30-20-10 0102030405060

(c)

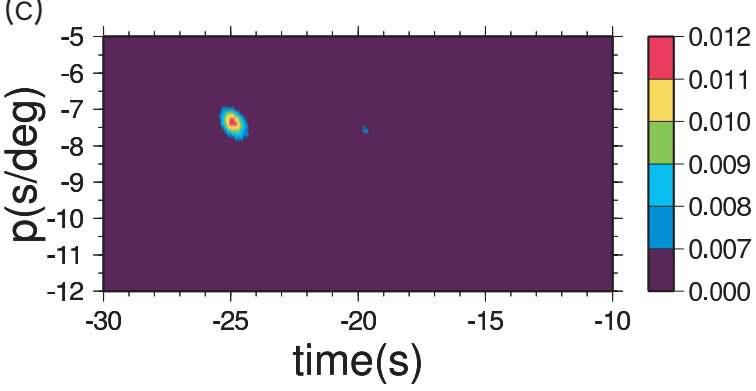

Figure 2: (a) Example of blocks and sub-blocks for stacking analysis. The star represents the location of the reference point for stacking, (b) pre-stacked waveforms in each subblock aligned with the arrival time of $S c S$ phase in red, and (c) amplitude contours of their slant stacking with the maximum signal corresponding to the dotted line in (b). 

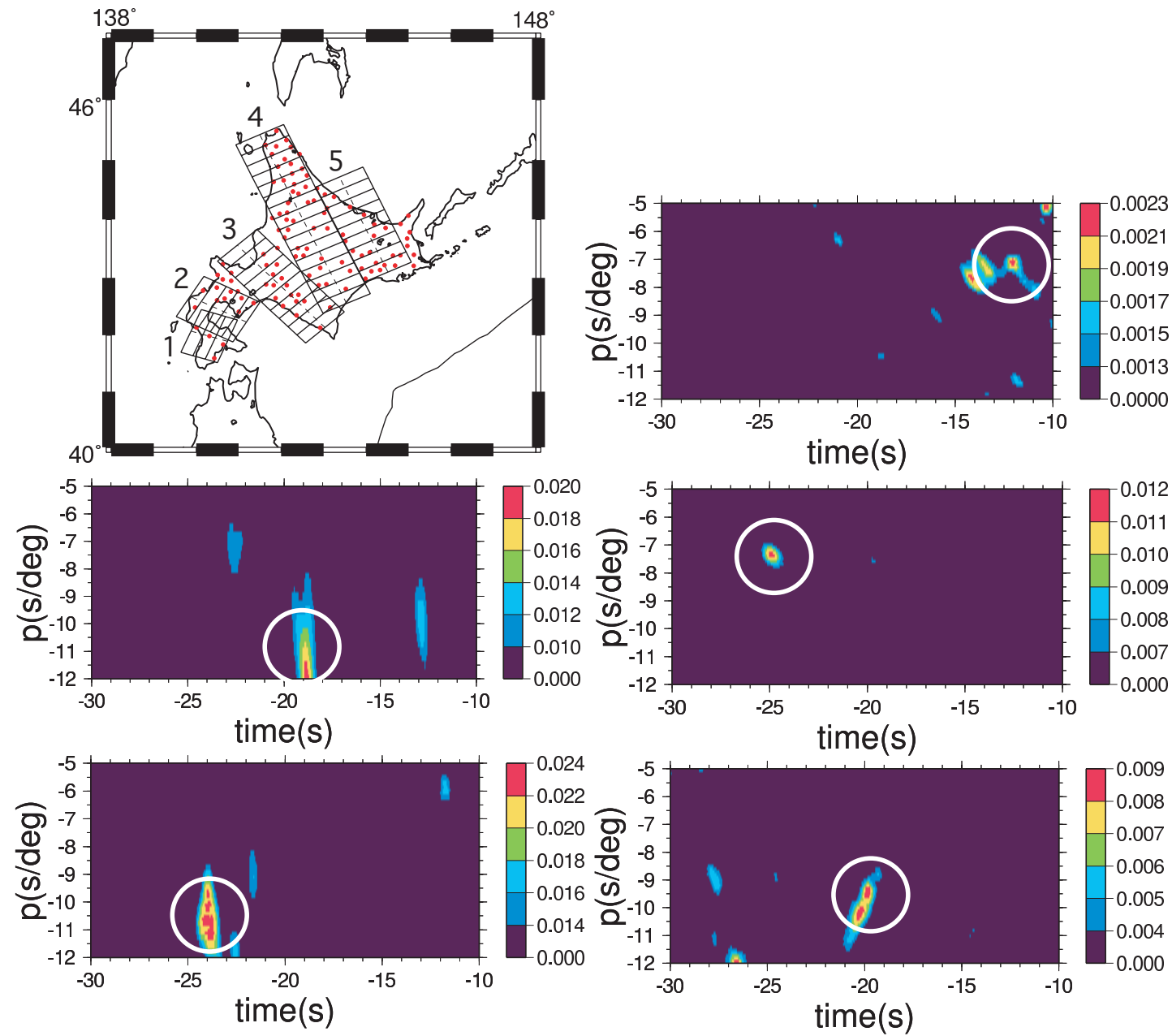

Figure 3: Maps of five blocks, and their amplitude contours of each block derived from the slant stack: blocks 1 and 2 in the left and blocks 3, 4 and 5 in the right. 
(a)

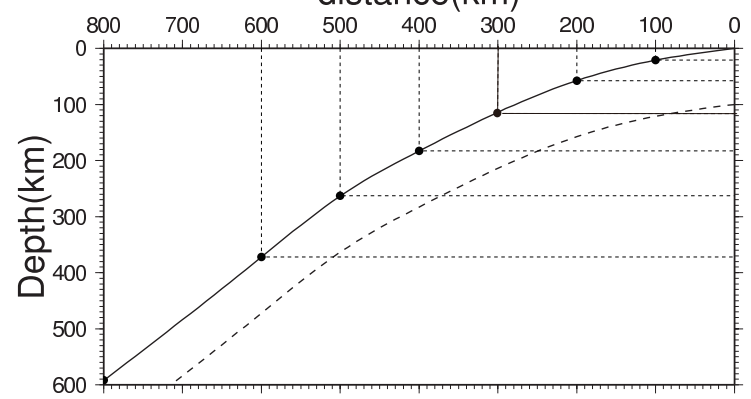

(b)

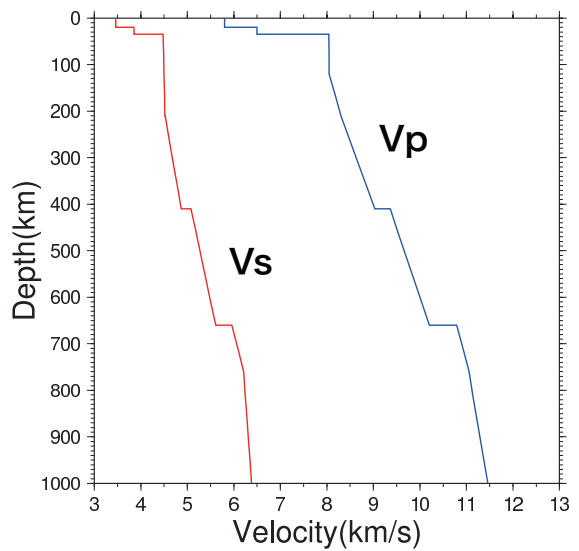

Figure 4: (a) Seven parameters for the plate model, and (b) reference velocity model of ak135. 


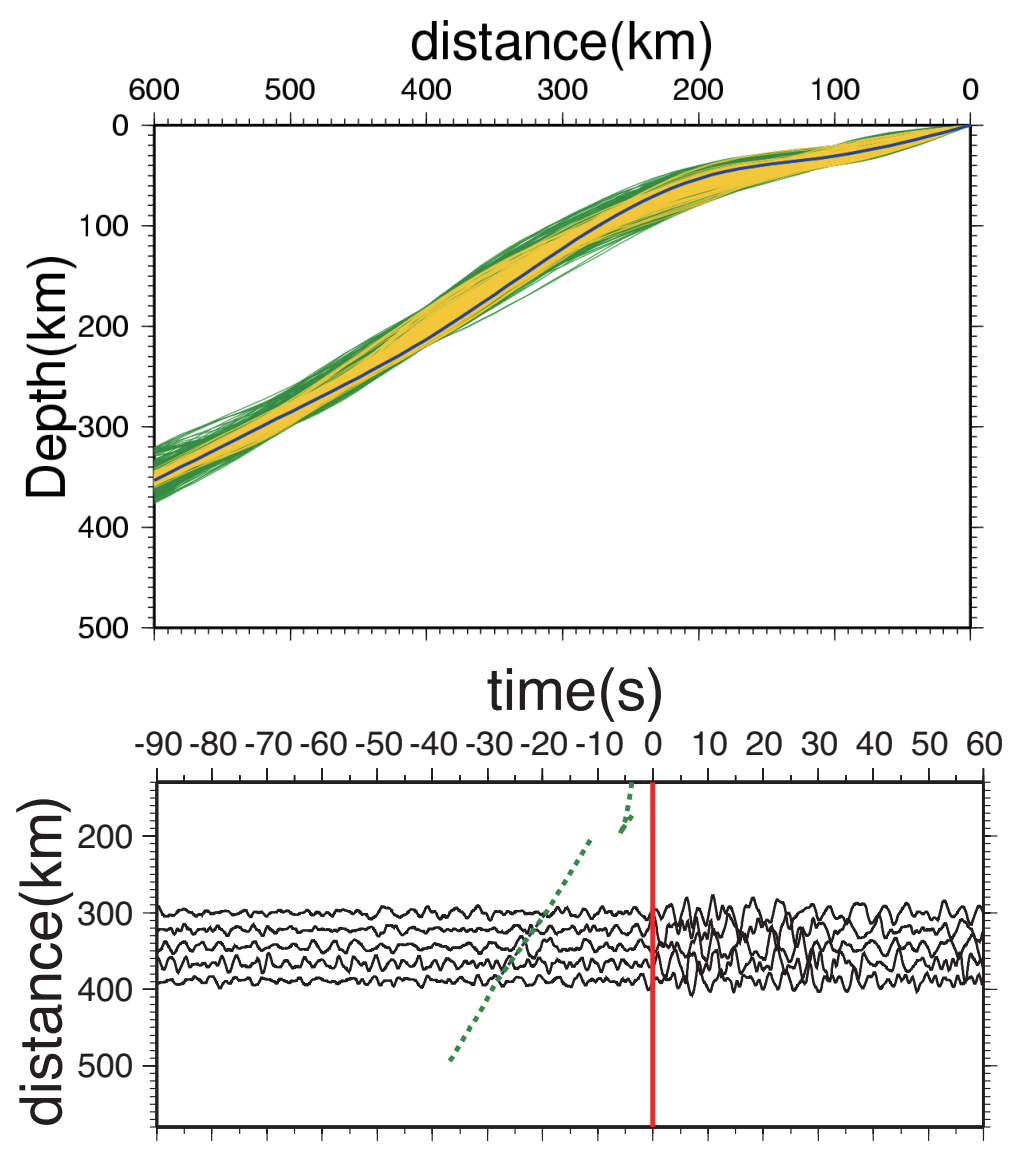

Figure 5: Total of the 6,500 plate models and the optimal model (thick blue line) derived from the model parameter search with the Neighbourhood Algorithm, together with waveforms to be stacked for block 2. All the plate models are ranked in order of the smaller misfit, and are plotted with colors varying from green (larger misfit) to yellow (smaller misfit). For the optimal model, waveforms are stacked along the dotted line. 

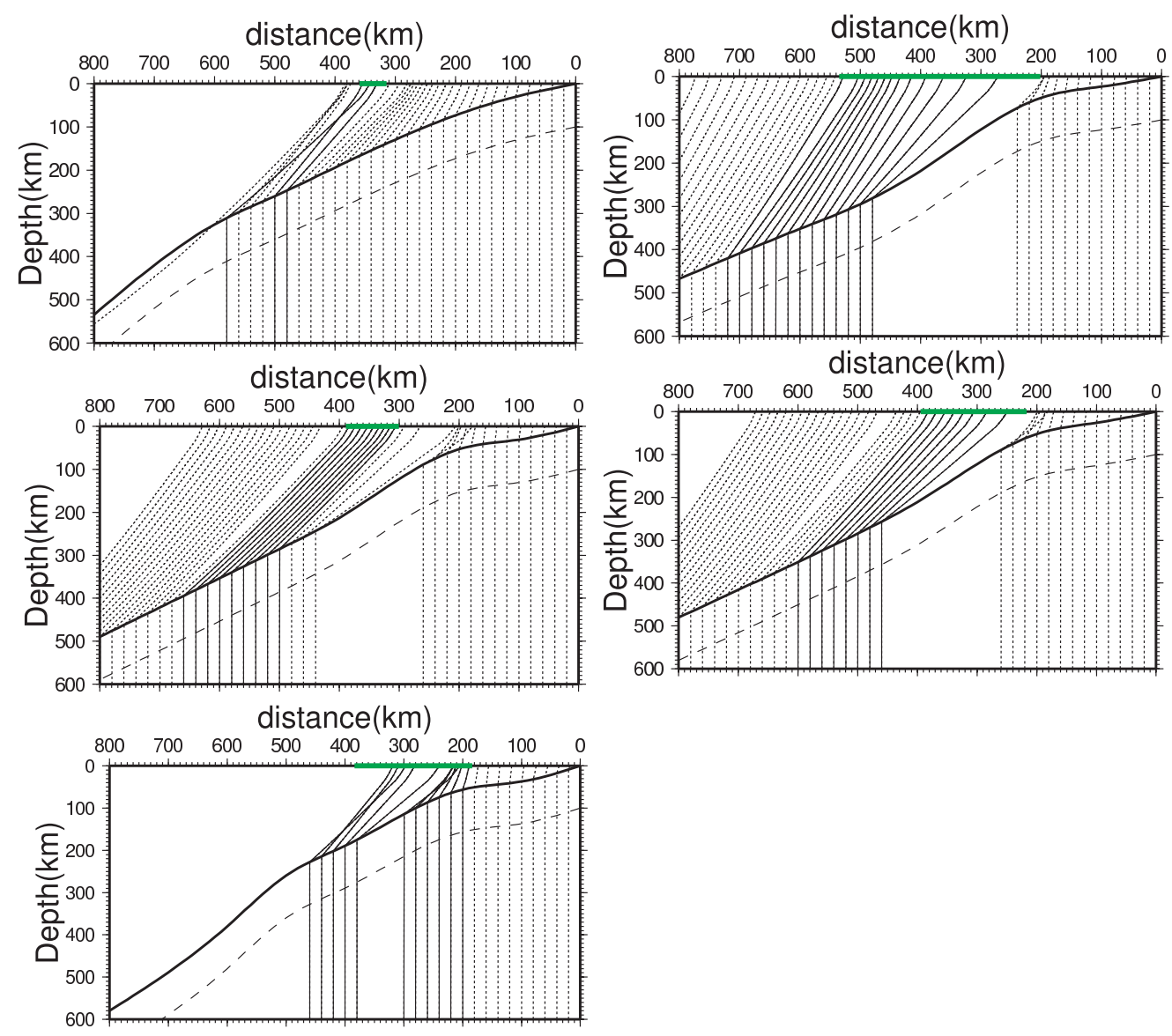

Figure 6: Optimal plate models and ray paths of $S c S p$ phases for blocks 1, 2, and 3 in the left, and blocks 4 and 5 in the right. 


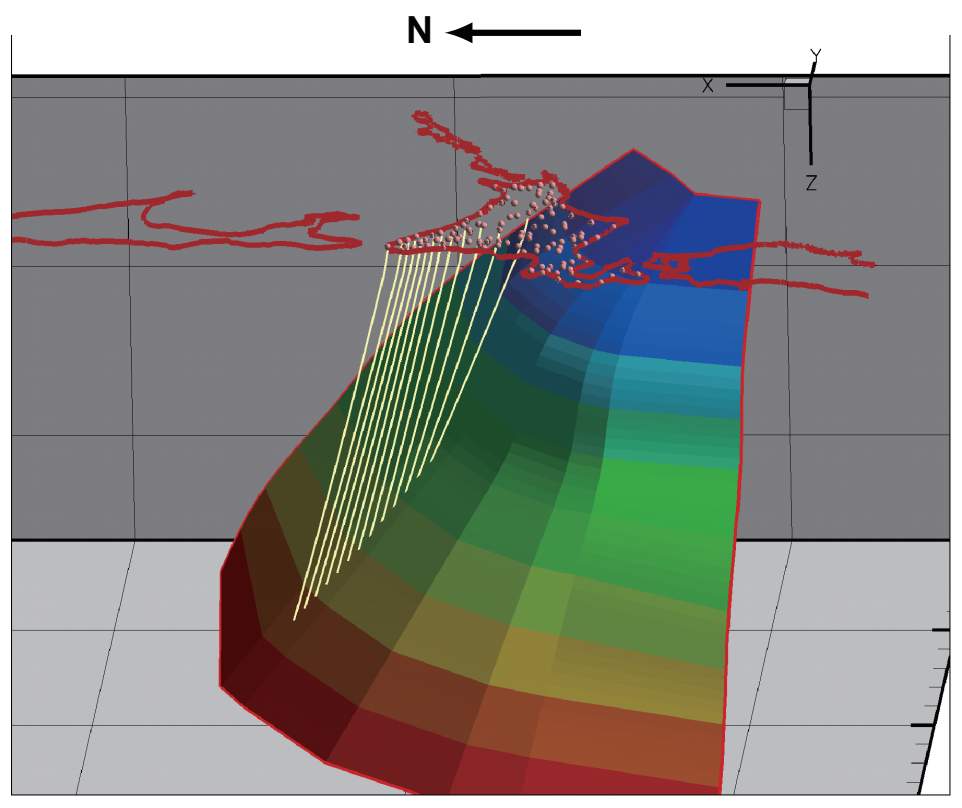

Figure 7: Three-dimensional view of the inverted upper boundary of the Pacific plate estimated from the 2-D five plate models in Figure 6 with a cubic spline function, and ray paths of $S c S p$ for block 4 . 


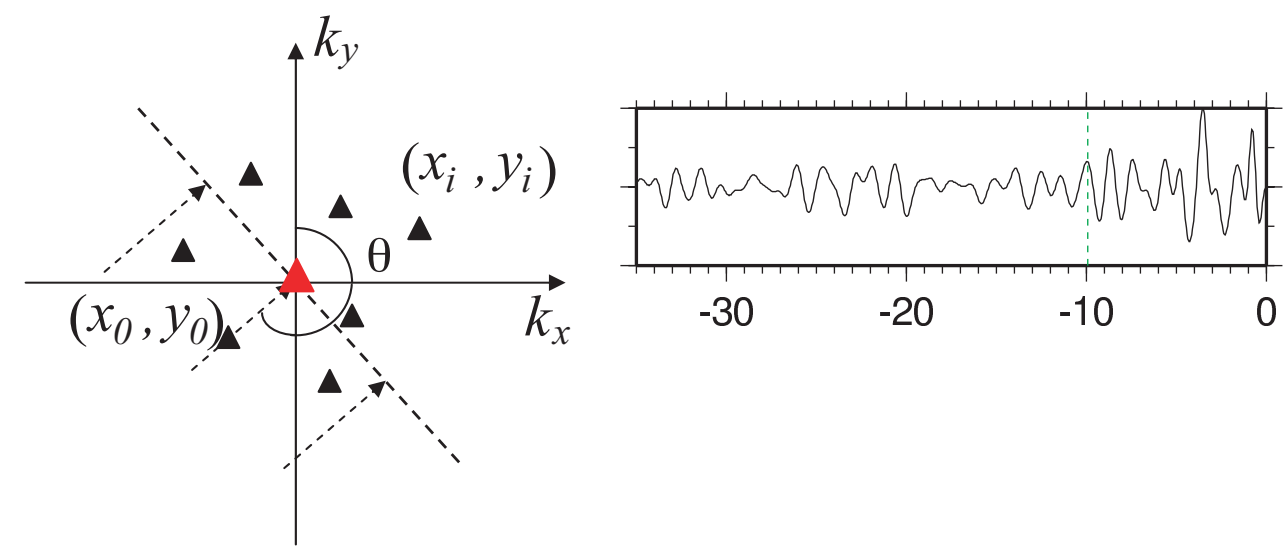

Figure 8: Schematic view of $f$ - $k$ spectral analysis. A plane-wave is incident to the reference station located at $\left(x_{0}, y_{0}\right)$ with adjacent stations at $\left(x_{i}, y_{i}\right)$, and an example of beam outputs with the corresponding $S c S p$ phase represented by the dashed line. 

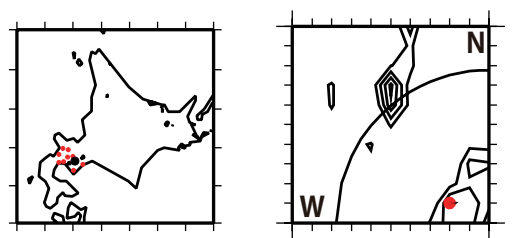

OHTH
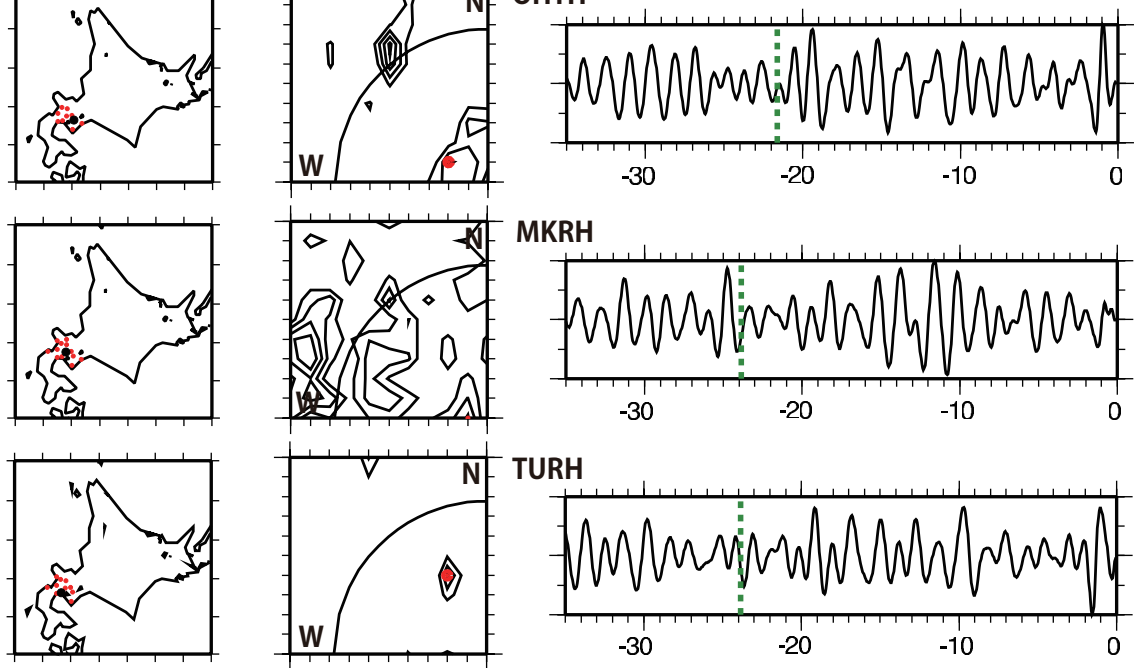

MKRH
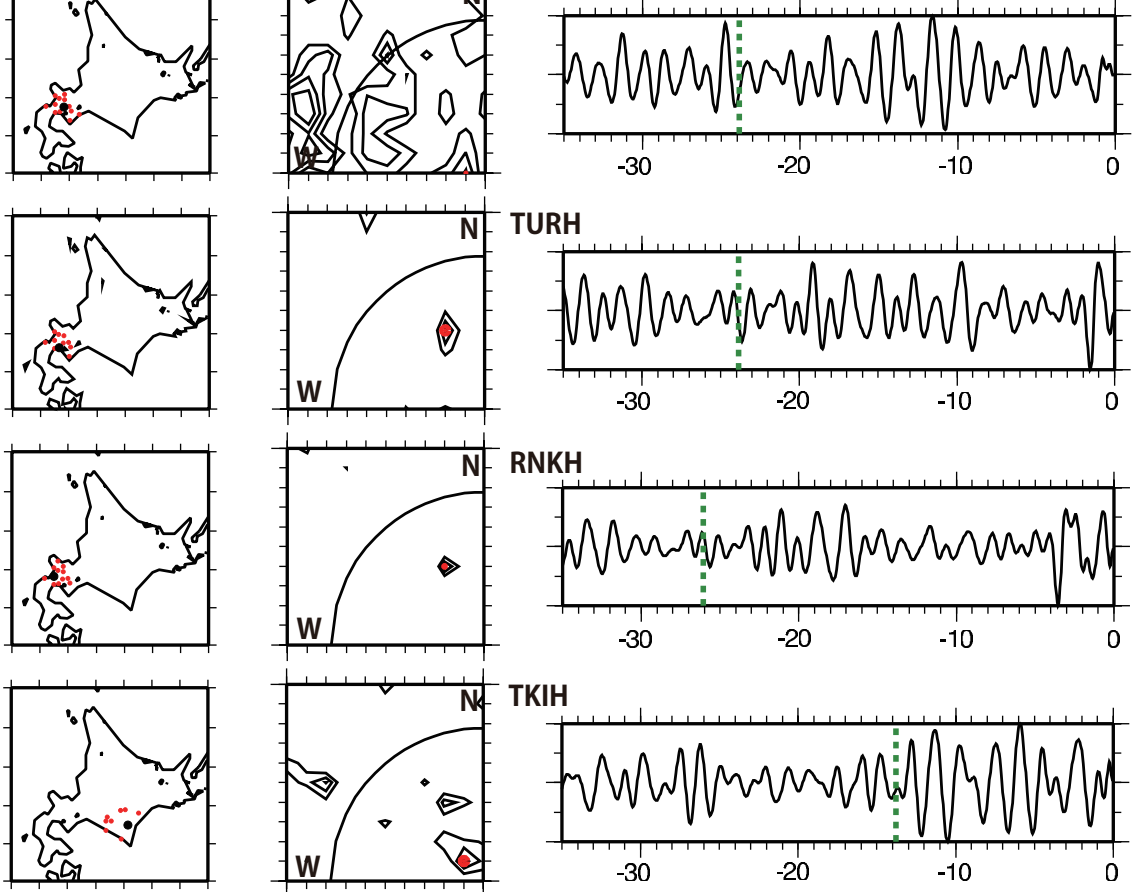

TKIH
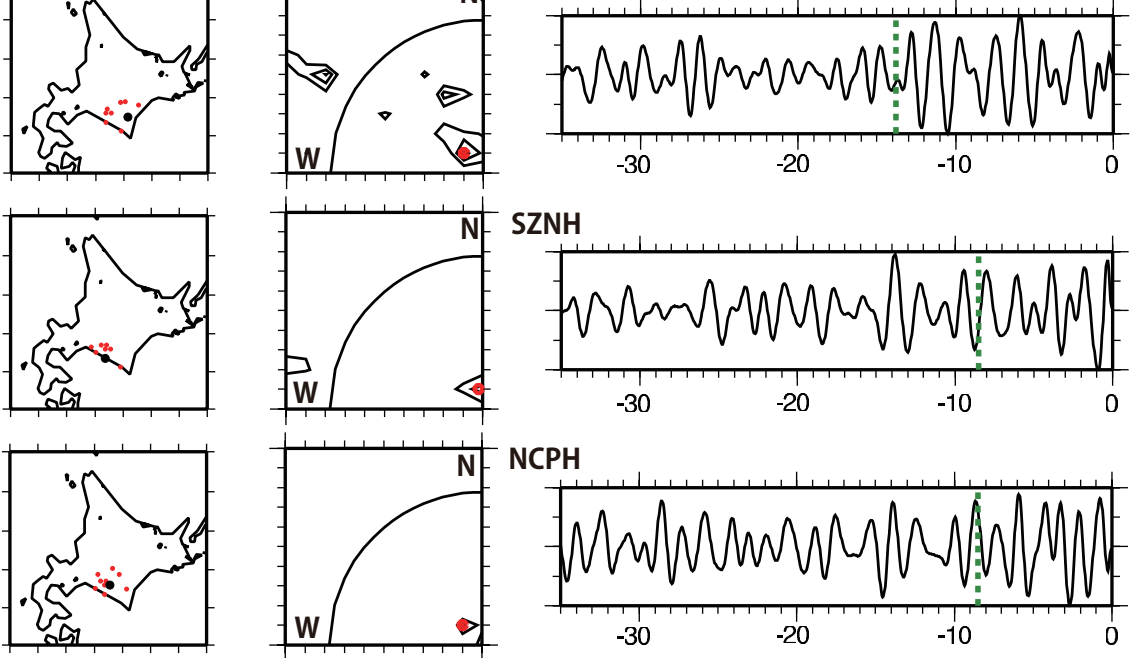

SZNH

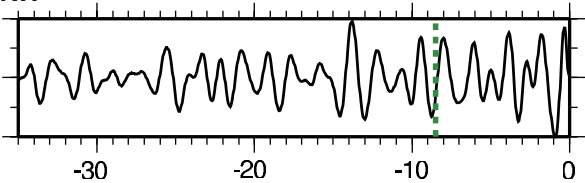

Figure 9: Examples of $f-k$ spectral analysis. The reference and adjacent stations in the left, contours of power spectra in the wavenumber domain in the center, and corresponding beam outputs in the right with the dotted lines as the estimated $S c S p$ arrivals. 


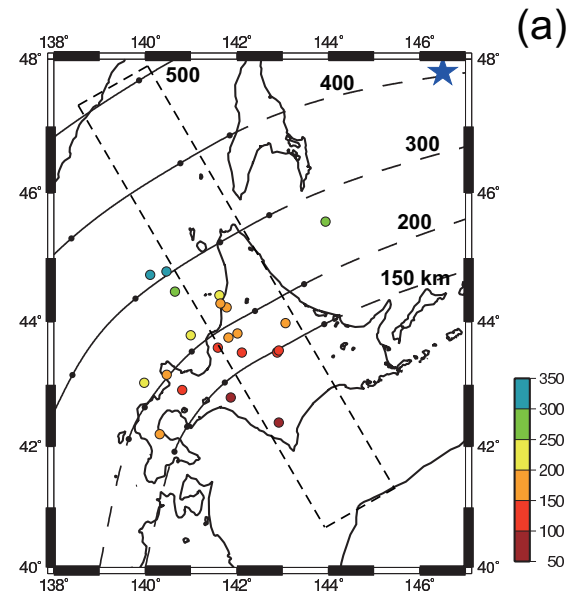

(a)

Figure 10: (a) Comparison of the contours for depths of the upper plate boundary derived from the NA algorithm with the conversion depths of $S c S p$ phase estimated by the $f-k$ spectral analysis, and (b) plate geometry in the region represented by the block of (a) with revision of the conversion depths derived from the $f-k$ spectral analysis, together with its earthquake locations obtained by Katsumata et al. (2003). 